\title{
Are the most metal-poor galaxies young?
}

\author{
Daniel Kunth $^{1}$ and Göran Östlin ${ }^{2}$ \\ ${ }^{1}$ Institut d'Astrophysique de Paris, 98bis Boulevard Arago, F75014 Paris, France \\ email: kunth@iap.fr \\ ${ }^{2}$ Stockholm observatory, AlbaNova University Center, 10691 Stockholm, Sweden \\ email: ostlin@astro.su.se
}

\begin{abstract}
We review the possibility that metallicity could provide a diagnostic for the age of a galaxy, hence that the most metal-poor star forming galaxies in the local universe may be genuinely young. Indeed, observational evidence for "downsizing" shows the average age of the stars in a galaxy to decrease with decreasing mass and metallicity. However, we conclude both from observational and theoretical viewpoints that metallicity is not an arrow of time. Consequently the most metal-poor galaxies of our local universe are not necessarely young. Current observations suggest that an old stellar population is present in all metal-poor galaxies, although a couple of cases, e.g. IZw18, remain under debate. Further observations with more sentitive equipement should settle this question in the coming years.
\end{abstract}

Keywords. galaxies: abundances, dwarf, evolution

\section{What is a young galaxy?}

In view of the recent popularity of the downsizing scenarios, in which mean stellar age and metallicity decrease with decreasing galactic mass it might be tempting to conjecture that some metal-poor, actively star forming dwarf galaxies may be genuinely young, now forming their first generation of stars. On the other hand, some of the most metal-poor galaxies in our vicinity are found among the dSph satellites of the Milky Way, which have old stellar populations. Hence, it is quite obvious that metallicity cannot be used as a simple cosmic clock, and we can straightforwardly answer the title question of this invited talk with a firm No. However, the bare possibility that some local galaxies may be young calls for a little more scrutiny in answering to this question.

What criteria must be met before we decide that a clump of matter is to be classified as a galaxy? Even if galaxies mostly consists of dark matter, we classify them from their stellar population and are inclined towards using stars and the star formation history as the relevant temporal measure for galaxy formation. Galaxies do not live in isolation: they merge, tear out gas and stars from each other, and accrete matter. What is the proper age definition for a galaxy in this context? We take here the working definition that the age of a galaxy is equal to that of its oldest stars.

This subject has been reviewed by Kunth \& Östlin (2000) and Kunth \& Östlin (2001), and we reiterate one conclusion from this work: finding a genuinely young galaxy would be such an interesting discovery that the burden of proof must lie on the youth hypothesis. Only when it has been shown beyond reasonable doubt, the limit being set by current technology, that a galaxy contains no old stars should we term a galaxy young.

\section{Which are the most metal-poor galaxies?}

Among local actively star forming galaxies, most are dwarfs: dwarf irregulars (dIrr) and Blue Compact Dwarfs (BCD). They remain, however, a minority among the general 
population of dwarf galaxies, Kunth \& Östlin (2000). At optical wavelengths, their spectra are dominated by young stars and ionised gas, closely resembling those of giant HII regions in nearby spiral and irregular galaxies. Analysis of their spectra show that most of them are metal deficient. Towards the lower end of the metallicity distribution $\left(\mathrm{O} / \mathrm{H} \sim 1 / 50 Z_{\odot}\right)$ we find galaxies like IZw18 and SBS0335-052. Due to their extreme properties it has been conjectured by Sargent \& Searle (1970) that these chemically unevolved galaxies could be young systems still in the proceess of forming. However, dwarf spheroidal galaxies (dSph) can be even more metal-poor, if judged by $[\mathrm{Fe} / \mathrm{H}]$ for the stellar population, but we should keep in mind that this is not directly comparable to nebular [O/H]-Grebel et al. (2003). Yet, dSphs are undisputably old.

Since metal-poor galaxies exist and are common at low redshift, one could of course expect many more at high redshifts. The first generation of star forming galaxies must, by definition, have been both young and metal-poor. However, selection effects tend to discriminate against the smallest, likely most metal-poor, galaxies in the distant universe, and the rather few estimates for Lyman Break Galaxies point to rather 'normal' abundances (say $1 / 3$ to $Z_{\odot}$ ) - Pettini (2006).

We note that many parameters control the observed metallicity in a given galaxy. They are incorporated into chemical evolutionnary models and include : stellar evolution and nucleosynthesis, inflows and outflows and the problem of the mixing and dispersion time scale of freshly released heavy elements. Kunth \& Östlin (2000) caution the use of simple classical closed box model although clearly, gas mass fraction seems to play a major role Lequeux et al. (1979). Moreover metallicity measurements may be relevant to only one particular component of a galaxy. A suggestion of Kunth \& Sargent (1986) has been made that HII gas could enrich itself with metals expelled by CC-SNe of time scales shorter than the life time of a starburst. Recent FUSE observations of some dwarf galaxies show a possible disconnect in metallicity between HI and HII regions - Cannon et al. (2005), Lebouteiller et al. (2006) - although possible saturation effects on the line of sight may alter such a comparison - Lebouteiller et al. (2006).

\section{Metallicity as an arrow of time}

The metal content of galaxies being an important diagnostic of galactic evolution, the question is whether metallicity can be regarded as an arrow of time? A useful connection between evolutionary state and metallicity is the luminosity-metallicity relationship. Such a relation can naturally arise if smaller galaxies have larger gas mass fractions than larger galaxies, i.e. simply because low mass galaxies have been less efficient in forming stars and therefore are less evolved. Another more likely possibility - see Tremonti et al. (2004) - is that more massive galaxies can retain metals more easily than less massive galaxies because of their deeper gravitational wells, making them less susceptible to metal loss in galactic winds - Garnett (2002).

Numerical simulations by Cen \& Ostriker (1999) predicting the evolution of the metal content of the Universe show that metallicity is a stronger function of density than age; moreover with a considerable scatter. At low redshift, one would expect a few percent of the gas-rich dwarfs to have metallicity on the order of IZw18, without invoking their youth.

In the past, the scatter in the $\mathrm{N} / \mathrm{O}$ versus $\mathrm{O} / \mathrm{H}$ diagram had been considered to be larger than the observational uncertainties. Time delays between the production of oxygen due to massive stars and that of nitrogen were a likely part of the explanation although this point of view was challenged by Izotov \& Thuan (1999). Indeed their observations not only suggested a small intrinsic dispersion of $\log \mathrm{N} / \mathrm{O}( \pm 0.02 \mathrm{dex})$ at low 
metallicities but a similar behaviour was found for $\mathrm{C} / \mathrm{O}$ and some other ratios. They concluded that galaxies with such low abundances are genuinely young (less than $40 \mathrm{Myr}$ old), now making their first generation of stars. Moreover they claimed that all galaxies with $7.6 \leqslant 12+\log (\mathrm{O} / \mathrm{H}) \leqslant 8.2$ have ages from 100 to 500 Myr. However, independent data such as the CMDs suggest that these galaxies do in fact contain old stars and there are definitely many BCDs with $12+\log (\mathrm{O} / \mathrm{H})<8.2$ which have been demonstrated to be much older than $500 \mathrm{Myr}$ (see next section). Nevertheless, the scatter is surprisingly small considering the short time scale for the production of oxygen (as compared to iron production) because different stellar masses are involved. Now, more recent data using SDSS show a much larger increase of the scatter at low $(\mathrm{O} / \mathrm{H})$ - Izotov et al. (2006) while other interpretations for the $\mathrm{N} / \mathrm{O}$ behaviour are also possible. A similar pattern is seen for N/O observations of HiI regions in spirals - van Zee et al.(1998), and old low surface brightness galaxies - Rönnback \& Bergvall (1995); Bergvall et al. (1999), have $\mathrm{N} / \mathrm{O}$ comparable to those of the most metal-poor BCGs.

\section{Ages of metal-poor galaxies}

Colour-magnitude diagrams (CMDs) allows for the most direct determination of the age of a stellar population. It is important to point out that in all nearby galaxies where CMDs have been obtained to the depth required for a clear detection of the red giant branch, has this also been found, showing beyond doubt that these systems are not young

Following Izotov \& Thuan (1999) a galaxy such as IZw18, with its record low abundance $(12+\log (\mathrm{O} / \mathrm{H})=7.18)$, should be genuinely young. There are nevertheless independent evidence suggesting that IZw18 does indeed host an old underlying population (see M. Tosi, this volume). A careful analysis of archival HST/WFPC2 data (Aloisi et al.(1999)), as well as independent HST/NICMOS observations (Östlin (2000), Östlin \& Mouhcine (2005)), both give support for an age in excess of 0.5 Gyr. Using new, very sensitive, HST/ACS observations Izotov \& Thuan(2004) failed to trace an old RGB in IZw18, but a re-analysis of the same data by Momany et al.(2005) of IZw18 do suggest the presence of an older RGB with an age of several Gyrs.

In cases where galaxies are too distant for a CMD to be obtained, other ways have to be sought to estimate their age. One such method involves using star clusters - usually good representations of single stellar populations whose ages can be determined with less degeneracies than mixed stellar populations. Limitations are rather set by statistical effects (in dwarf galaxies suitable massive star clusters are rare). One example of a metalpoor galaxy whose age can be tied to $\sim 10$ Gyr is the luminous compact galaxy ESO 33804, a.k.a. Tol 1924-416 (Östlin et al. 1998).

As most active star formation in metal-poor galaxies tends to be centrally concentrated, a natural place to look for old stars are the outskirts, or "haloes" of e.g. BCDs by comparing optical and infrared colours to spectral synthesis models (e.g. Bergvall \& Östlin 2002). The further out one looks, the smaller the contamination from young stars, but also lesser is the light to analyse. A major problem is that of wide spread ionised gas. In addition, the ionised gas may have a different scale length from the stellar population (Papaderos et al. 2002), further complicating the analysis.

With good quality data it is even possible to date the old population in the centra of star forming galaxies, e.g. as has been performed with some SDSS spectra of star forming dwarfs (Corbin et al. 2006). 


\section{Outlook}

Our discussion lead us to conclude that no compelling observational evidence for any young local galaxy has been provided so far. Moreover metallicity is not an arrow of time, but a product of the past star formation history, combined with the mass-loss and accretion history. Only few local galaxies remain under debate but this is likely because they remain beyond sensitivity thresholds! With JWST we can probe a little further, but not much, e.g. getting a CMD for SBS 0335-052 will still be a tough task. Do we at all expect young galaxies in our Local Universe? Could some HI clouds that survived reionisation start to collapse now? Then they need to have been unable to form any stars early on but still be masssive enough not to boil off completely during reionisation. It appears unlikely that both requirements can be fulfilled.

\section{References}

Aloisi, A., Tosi, M., \& Greggio, L., 1999, AJ, 118, 302

Bergvall, N., \& Östlin, G., 1999, A\&A, 390, 891

Bergvall, N., R" onnback, J., Masegosa, J., \& Östlin, G., 1999, A\&\&A, 341, 697

Cannon, J. M., Skillman, E. D., Sembach, K. R., \& Bomans, D. J., 2005, ApJ, 618, 247

Cen R., \& Ostriker J.P., 1999, ApJ, 519, L109

Corbin M., Vacca W., Cid Fernandes R., et al., 2006, ApJ, in press.

Dufour, R. J., Garnett, D. R., Skillman, E. D., \& Shields, G. A., 1996, Science with the Hubble Space Telescope - II, 348

Garnett, D. R., 2002, ApJ, 581, 1019

Grebel, E. K., Gallagher, J. S., III, \& Harbeck, D., 2003, AJ, 125, 1926

Hunter, D. A., \& Thronson, H. A., Jr., 1995, ApJ, 452, 238

Izotov, Y. I., \& Thuan, T. X., 1999, ApJ, 511, 639

Izotov, Y. I., \& Thuan, T. X., 2004, ApJ, 616, 768

Izotov, Y. I., Stasińska, G., Meynet, G., Guseva, N. G., \& Thuan, T. X., 2006, ApJ, 448, 955

Kunth D., \& Östlin G., 2000, A\& $A R v, 10,1$

Kunth D., \& Östlin G., 2001, ApSSS , 277, 281

Kunth, D., \& Sargent, W. L. W., 1986, ApJ, 300, 496

Lebouteiller, V., Kunth, D., Lequeux, J., Aloisi, A., Desert, J. -., Hebrard, G., Lecavelier des Etangs, A., \& Vidal-Madjar, A., 2006, Astrophysics e-prints, astro-ph/0608445

Lequeux, J., Maucherat-Joubert, M., Deharveng, J. M., \& Kunth, D., 1981, A\&A, 103, 305

Lequeux, J., Peimbert, M.; Rayo, J. F.; Serrano, A.; \& Torres-Peimbert 1979, A\&A, 80, 155

Momany, Y., et al., 2005, ApJ, 439, 111

Östlin, G., Bergvall, N., \& Roennback, J., 1998, A\& $A, 335,85$

Östlin G., 2000, ApJ, 535, L99

Östlin G., \& Mouhcine, M., 2005, A\&\&A, 433, 979

Papaderos P., Izotov Y., Thuan T., et al., 2002, A\&GA, 393, 461

Pettini, M., 2006, ASP Conf. Ser., 353, 363

Rönnback, J., \& Bergvall, N., 1995, A\&A, 302, 353

Sargent, W. L. W., \& Searle, L., 1970, ApJ, 162, L155

Tremonti, C. A., et al., 2004, ApJ, 613, 898

van Zee, L., Salzer, J. J., \& Haynes, M. P., 1998, ApJ, 497, L1 